\title{
Desenvolvimento e Avaliação do Jogo DuchsVille para Apoiar o Processo de Aprendizagem Nutricional: estudo de caso com adolescentes com distrofia muscular de Duchenne
}

\author{
Ana Grasielle Dionísio Corrêa ${ }^{1}$, Adriana Nathalie Klein ${ }^{2}$, Cristina Salvioni ${ }^{2}$, Irene \\ Karaguilla Ficheman ${ }^{1}$, Roseli de Deus Lopes ${ }^{1}$ \\ ${ }^{1}$ Laboratório de Sistemas Integráveis da Escola Politécnica (LSI-EP) \\ Universidade de São Paulo (USP)
}

Av. Prof. Luciano Gualberto, travessa 3, nº 158, CEP: 05508-970, São Paulo/SP, Brasil

2 Associação Brasileira de Distrofia Muscular (ABDIM)

Rua Engenheiro Teixeira Soares, 715 - Butantã - CEP:05505-030 - São Paulo- SP

\begin{abstract}
Resumo. O presente estudo teve como objetivo criar, implementar e avaliar um jogo eletrônico para educação alimentar e nutricional de crianças com distrofia muscular de Duchenne (DMD). Participaram do estudo, 27 profissionais da saúde e 10 adolescentes com DMD. Foram aplicados questionários de pré-teste a fim de coletar informações a respeito dos conhecimentos prévios sobre hábitos alimentares e pós-teste para analisar os ganhos de informações após o jogo. Observou-se que as crianças retiveram conhecimento sobre alimentação e nutrição após jogo. Concluiu-se neste trabalho que a interatividade proporcionada pelos jogos eletrônicos, pode estimular e favorecer na construção do conhecimento sobre nutrição e hábitos alimentares saudáveis.
\end{abstract}

Palavras-chaves: jogos educacionais, educação nutricional, distrofia muscular de Duchenne

Abstract. The present study aimed to create, implement and evaluate an electronic game for food and nutrition education of children with Duchenne Muscular Dystrophy (DMD). Ten seven health professionals and fifteen teenagers with DMD participated in the study. Pre-test questionnaires were used in order to collect information about prior diet knowledge and post-test questionnaires were applied after the game to analyze concept learning. We observed that children retained knowledge about food and nutrition after playing the game. We conclude in this study that games' interactivity can encourage and foster knowledge construction about nutrition and healthy eating habits.

Keywords: educational games, nutrition education, Duchenne muscular Dystrophy 


\section{Introdução}

A desnutrição, o excesso de peso e a obesidade são caracterizados pelo Comitê Permanente de Nutrição (Standing Committee on Nutrition 2006) como transição nutricional. Diversas pesquisas têm mostrado que o Brasil, assim como outros países em desenvolvimento, convivem com a transição nutricional, frequentemente determinada pela má-alimentação (Coutinho et al. 2008). Apesar da redução contínua dos casos de desnutrição no Brasil, são observadas prevalências crescentes de excesso de peso e obesidade, contribuindo com o aumento das doenças crônicas não transmissíveis. Um estudo feito, em 2009, pela Pesquisa de Orçamentos Familiares, realizada em parceria entre o Instituto Brasileiro de Geografia e Estatística (IBGE) e o Ministério da Saúde, constatou que o excesso de peso e a obesidade são encontrados com frequência, a partir de 5 anos de idade, em todos os grupos de renda e em todas as regiões brasileiras.

De acordo com Davidson \& Truby (2009), a obesidade e a desnutrição estão entre as complicações clínicas ou doenças mais frequentes associadas à distrofia muscular de Duchenne $\left(\mathrm{DMD}^{1}\right)$, ocorrem em cerca de $54 \%$ dos casos, afetando diretamente a função respiratória e a habilidade para realizar as atividades de vida diária. Conforme Davidson e Truby (2009) e Santos et al. (2006), a obesidade pode ocorrer devido à superproteção da família, que permite excessos alimentares, mas ocorre principalmente pela diminuição da mobilidade e aumento da dependência, que prejudica a biomecânica da coluna vertebral e compromete o bom funcionamento das funções digestivas e respiratórias. Já a desnutrição, causa perda excessiva de peso, podendo diminuir a força dos músculos respiratórios e levar a uma insuficiência respiratória. Nos estágios finais da DMD, a desnutrição pode estar relacionada principalmente com a fraqueza e incoordenação dos músculos responsáveis pela mastigação e deglutição.

Segundo Santos (2005), a educação nutricional visa melhorar o estado nutricional, além de ter um papel importante em relação a promoção dos hábitos alimentares saudáveis, eliminação de práticas dietéticas insatisfatórias, introdução de práticas de higiene da alimentação, prevenção de doenças e uso mais eficiente dos recursos disponíveis para alimentação. Contudo, a DMD pode comprometer também os mecanismos e capacidades cognitivas necessárias à adequada educação nutricional (Vechia, 2009). Como se trata de uma doença crônica e de caráter progressivo, sendo as primeiras manifestações clínicas presentes a partir dos três anos de idade, as ações de orientação se tornam complicadas (Davidson; Truby 2009).

Refletindo sobre as experiências compartilhadas na prática assistencial com pessoas com DMD e, sobretudo, a respeito das dificuldades de adesão a uma alimentação adequada, surgiu o interesse em criar algo atrativo, dinâmico e interativo para apoiar esse processo. Este artigo descreve o desenvolvimento e a avaliação do jogo DuchsVille onde são abordadas as principais questões relacionadas à educação

\footnotetext{
${ }^{1}$ A Distrofia Muscular de Duchenne (DMD) é uma doença hereditária caracterizada pela progressiva e irreversível degeneração da musculatura esquelética, resultando em uma fraqueza muscular generalizada, em consequência da mutação do cromossomo XP21, fator que limita a produção da distrofina, uma proteína essencial para a função do músculo estriado. Trata-se de uma patologia de herança recessiva que afeta o gênero masculino com uma incidência de 1(um) em cada 3.500 nascidos vivos, (Manzur e Muntoni 2009).
} 
nutricional de crianças com DMD. Além do capítulo Nutrição, o Jogo DuchsVille possui mais outros 3 capítulos: Reabilitação, Intervenção Respiratória e Área Clínica que não serão abordados neste artigo.

\section{Referencial Teórico}

\subsection{A Educação Nutricional de Crianças com DMD}

O tratamento nutricional, de crianças com DMD, deve ser adaptado a cada estágio da doença. Isso porque, por volta dos 13 anos de idade quando acontece a perda de marcha e dependência da cadeira de rodas, concomitante à substituição de fibras musculares, (fonte de consumo energético por tecido adiposo), os individuos aumentam abruptamente seu peso corporal (Hankard et al. 1996). Nessa fase, deve-se evitar o ganho excessivo de peso por meio de orientações nutricionais e aconselhamento de restrição energética, especialmente fontes de gorduras e/ou açucares. Nessa situação, é comum observar pais e/ou cuidadores relutando em reduzir alimentos, tais como doces e refrigerantes muitas vezes administradas como forma de compensação.

Já em fases tardias da doença, o que necessita de atenção é a desnutrição. Dificuldade de deglutição, abrir a boca e mastigação levando a um aumento na duração das refeições, perda da capacidade de auto-alimentação, redução da ingestão alimentar podem contribuir com o quadro (Davidson; Truby 2009), (Pane et al. 2006). Modificar a consistência dos alimentos e adequar a dieta de acordo com a deglutição individual é uma importante estratégia para reduzir as dificuldades de alimentação.

Conforme Davidson \& Truby (2009), a combinação entre imobilidade e fraqueza muscular pode predispor a obstipação intestinal. Portanto, uma dieta com maior conteúdo de água e fibras deve ser recomendada. Frutas e vegetais são ricos em fibras e indicados para uma boa nutrição. Ainda segundo Davidson \& Truby (2009), crianças em tratamento com glicocorticoides, medicamento de uso comum na DMD, precisam receber maior restrição energética e eventualmente restrição da ingestão de sal (os glicocorticoides aumentam o apetite, causam deposição aumentada de gorduras e retenção de sal). Mayo et al. (2012), descrevem como doses farmacológicas de glicocorticóide orais podem suprimir a formação de osso e aumentar a reabsorção óssea, levando à osteoporose. Portanto, uma dieta rica em cálcio também deve ser observada.

Com indivíduos em uso de ventilação não invasiva os cuidados com a nutrição devem ser redobrados (Davidson; Truby 2009). Uma dieta rica em carboidratos leva ao aumento da pressão parcial de gás carbônico no sangue por excesso de produção, dificultando o controle do aparelho de respiração. Neste caso, é muito importante avaliar as necessidades energéticas e orientar a dieta que atenda a todos os problemas que o indivíduo apresente (um profissional da área é imprescindível).

\subsection{A Importância dos Jogos Eletrônicos para a Educação Nutricional}

De acordo com Jorge e Perez (2004), somente dialogar com jovens sobre a importância da alimentação saudável tem se tornado uma tarefa cada vez mais difícil nos últimos anos. Segundo os autores, é necessáro aproximar o público, conhecê-lo, falar sua linguagem e o mais importante: ilustrar. Pesquisas mostram que, quando recursos tais como videos, músicas, figuras, jogos, entre outros são utilizados como recursos 
ilustrativos, o aprendizado se torna mais agradável. Conforme Savi e Ulbricht (2008), os jogos são estratégias didáticas significativas para a educação em saúde, pois trabalham a sensibilidade e as emoções dos envolvidos, possibilitando o autoconhecimento. Magalhães (2007), descrevendo sobre a utilização de jogo com crianças de 7 a 14 anos, afirmou que este se mostrou como uma alternativa educativa viável, por sua natureza lúdica e informativa e por seu comprometimento com a interação humana. Em outro estudo, Toscani et al. (2007) constataram que o jogo educacional é uma ferramenta lúdica que faz do participante um agente ativo no processo educacional.

Baranowski et al. (2003) realizou um estudo randomizado controlado que objetivou demonstrar, por meio de um jogo multimidia, melhoras nos hábitos alimentares crianças da $4^{\mathrm{a}}$ série do ensino fundamental, principalmente para o consumo de frutas, sucos e legumes. O jogo foi aplicado em 26 escolas do ensino fundamental, totalizando 1.578 crianças. 10 sessões de 25 min psicoeducacionais, com apoio do jogo, foram realizadas durante 5 semanas consecutivas. Baseado na Teoria Social Cognitiva, o jogo buscou aumentar preferências por frutas, sucos e legumes, por meio da associação da diversão ao seu consumo tais como, aperfeiçoar as habilidades para preparar refeições baseadas em frutas, sucos e legumes por meio de cardápios virtuais. Dois grupos (Controle e Experimental) foram formados aleatoriamente e a eles oferecidos almoços gratuítos ou à preços baixos. Os dados foram coletados por recordatório alimentar antes e após o jogo e, posteriormente analisados por meio de testes estatísticos. O Grupo Experimental (GE) recebeu intervenções psicoeducativas com apoio do jogo, ao contrário do Grupo Controle (GC). Os autores constataram que o GE consumiu mais frutas e vegetais no pré-teste e aumentou o consumo de frutas, sucos e vegetais após o jogo. Os autores concluíram, por meio desta pesquisa, que os jogos de multimídia psicoeducativa têm potencial de mudar comportamentos e hábitos alimentares. Outras pesquisas relacionadas ao uso de jogos na prática educativa em saúde alimentar foram realizadas por Zaccarelli et al. (2000); Costa et al. (2004); Barbosa (2010); Neves (2010).

\section{Desenvolvimento e Avaliação do Jogo DuchsVille}

\subsection{Análise dos Requisitos}

Como recomenda Preece, Rogers \& Sharp (2005), na etapa de levantamento de requisitos, foram conduzidas algumas seções de braimstorm com usuários finais a fim de coletar informações pertinentes ao conteúdo a ser abordado no jogo. Para tanto, foram entrevistados 27 profissionais com formação mínima de especialista ou mestre e com pelo menos dois anos de experiência no atendimento de pessoas com Distrofia Muscular (2 Geneticistas; 2 Médicos especializadas em Clínica Médica; 3 Fisioterapeutas aquáticos; 7 Fisioterapeutas motores; 2 Fisioterapeutas respiratórios; 2 Assistentes Sociais; 2 Fonoaudiólogos; 1 Nutricionista; 3 Terapeutas Ocupacionais; 2 Pedagogos e 1 psicopedagogo) e 30 pacientes com diagnóstico de DMD. Um moderador (Terapeuta Ocupacional) e um observador (Psicopedagogo) lançaram a seguinte pergunta aos entrevistados: "Quais informações a cerca da DMD você considera mais importantes e que devem ser transmitidas constantemente aos portadores da doença?”. Todos os dados coletados foram anotados e transcritos em tabelas. Os dados coletados foram analisados e separados em quatro capítulos: Nutrição, Reabilitação, Intervenção 
Respiratória e Área Clínica. O critério para esta classificação foi a frequência destas citações nos discursos dos entrevistados.

\subsection{Análise da Viabilidade Técnica}

Foi feita uma análise de quais tecnologias poderiam ser utilizadas para a implementação do jogo, levando em consideração, o requisito do jogo ter "alta disponibilidade" requerido pelos profissionais na fase de análise de requisitos. Para tanto, foi elencada a ferramenta Adobe Flash CS5, pois possibilita o acesso do jogo pela Internet por meio de um navegador web com FlashPlayer instalado. A programação do jogo (linkagem das telas, animações e interações com o usuário) foi criada na linguagem de programação ActionScript 3.0 (AS3), suportada pelo Adobe Flash CS5. As ilustrações foram feitas com uso de uma mesa digitalizadora Intuos PTK-440. Para ilustração e design dos desenhos foram usados os softwares Adobe Photoshop e Adobe Illustrator que possibilitam exportar arquivos no formato suportado pelo Adobe Flash (TIF). Alguns vetores foram exportados em PNG, formato escolhido por suportar transparência.

\subsection{O Capítulo Nutrição}

O Jogo DuchsVille, capítulo Nutrição, tem por objetivo estimular, de forma interativa, a escolha por alimentos considerados saudáveis, focando principalmente na melhoria da alimentação e seus benefícios para a saúde. O jogo é indicado para crianças e adolescentes com DMD. Pode ser utilizado por qualquer outra pessoa, a partir do primeiro ciclo do ensino fundamental, como recomendado por Bizzo \& Leder (2005).

O Jogo possui dois desafios: Compras (Figura 1a) e Coleta Saudável (Figura 1b). O primeiro é caracterizado por Tarouco et al (2004) como um jogo lógico, pois estimula mais o raciocínio do que os reflexos. Nesse desafio, o jogador não tem limite de tempo e pode navegar pelo cenário em busca da solução para o problema. O segundo é caracterizado, também por Tarouco et al (2004), como jogo de ação, pois auxilia no desenvolvimento psicomotor, estimulando os reflexos e a coordenação motora. Nesse tipo de jogo, o jogador deve reagir com atenção, reforçando "repetindo" o conteúdo abordado no primeiro jogo.
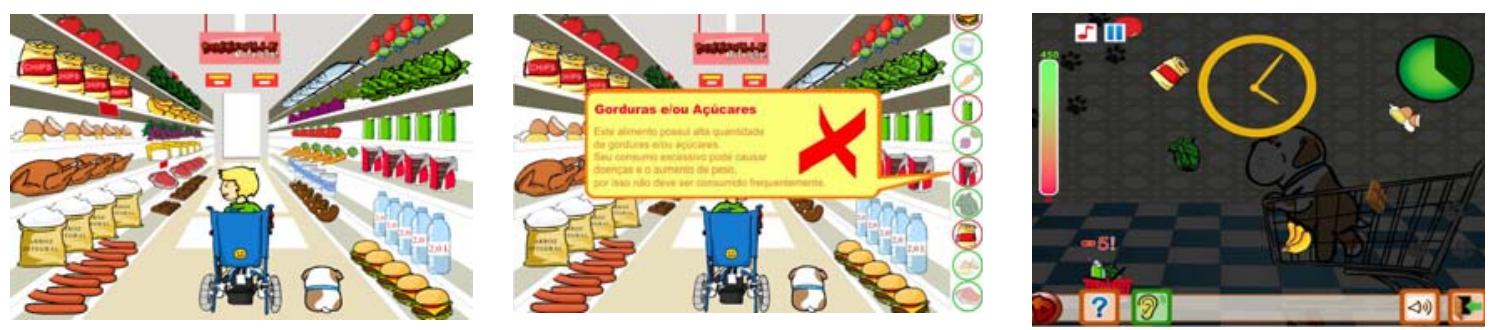

Figura 1 - a) Compras; b) Descrição dos nutrientes; c) Coleta Saudável

No caso do desafio das Compras, o jogador se depara com um cenário de um supermercado. Seu objetivo é selecionar 10 alimentos que devem ser consumidos diariamente para manter uma dieta equilibrada. Áudios narrados são disparados à medida que escolhas boas e ruins são realizadas, instruindo sobre a influência dos macro e micronutrientes encontrados em cada alimento. Por exemplo, no caso da escolha pelo chocolate, aparece a mensagem "Gorduras e/ou Açucares”, com a descrição das consequências trazidas ao organismo caso seja consumido diariamente (Figura 1b). Caso 
o jogador não tenha selecionado ao menos 8 dos 10 alimentos saudáveis, ele deve retornar para a tela da gôndola e substituir os alimentos não saudáveis por alimentos mais saudáveis. O jogo então estimula a observação e a reflexão ao fazer com que o jogador pense em quais alimentos poderia lhe trazer benefícios ou malefícios para sua saúde, caso seja consumido diariamente.

No caso do desafio Coleta Saudável, o jogador tem 1 (um) minuto para coletar, com uma cesta de supermercado, o maior número de alimentos considerados saudáveis (escolhidos na gôndola do desafio anterior). Este desafio é uma forma de reforçar o conteúdo apresentado no primeiro desafio. As imagens dos alimentos vão surgindo no topo da tela e vão caindo com velocidade progressiva, tornando o jogo mais difícil. A cada acerto, o jogador acumula +5 pontos e a cada erro perde -5 pontos. A cada 5 alimentos saudáveis coletados sequencialmente, o cronômetro do jogo é congelado por 10 segundos, o que aumenta a chances do jogador acumular mais pontos. Para acessar e jogar o desafio Coleta Saudável, o jogador deve selecionar ao menos 8 alimentos saudáveis no desafio de Compras. Desta forma, a possiblidade de jogar o desafio Coleta Saudável se torna uma premiação do desafio de Compras.

Ambos os desafios possuem trilhas sonoras que combinam com o clima do jogo. O desafio das Compras possui um ritmo lento, no estilo de som ambiente, remetendo ao jogador a sensação de tranquilidade. Já o desafio da Coleta Saudável, possui um ritmo mais acelerado com tom divertido.

\subsection{Avaliação Realizada com Usuários}

Participaram deste estudo 10 adolescentes com DMD em tratamento na Associação Brasileira de Distrofia Muscular de Duchenne (ABDIM). A amostra é constituída, em sua maioria, por usuários na faixa etária de 13 a 17 anos, todos estavam em processo de escolarização no primeiro ciclo do Ensino Fundamental. Para a seleção desta amostra, foi utilizada uma das técnicas de amostragem não probabilística que se caracteriza por não incluir todos os indivíduos da população alvo (Casey 1992). Optou-se pela amostragem por objetivo que busca por sujeitos que possuem uma característica de interesse específico à pesquisa, neste caso, crianças com DMD em processo de aprendizagem atendidas no setor de Escolarização da ABDIM. O principal critério de seleção foi o de estar alfabetizado e na faixa etária estabelecida como público-alvo do jogo, ou seja, adolescentes a partir dos 12 anos de idade e ter disponibilidade para participação na pesquisa.

Para coleta de dados foi elaborado, pelos autores desta pesquisa, um questionário quali-quantitativo (Figura 2a) com objetivo de coletar o conhecimento prévio do usuário em relação aos alimentos que ele considera saudáveis para consumo diário. Esse questionário contém a seguinte pergunta "Quais alimentos você deve consumir diariamente para manter uma alimentação saudável?” e, como respostas, 24 figuras de alimentos e bebidas que são apresentados nos desafios "Compras" e "Coleta Saudável". Desses alimentos, 14 são considerados saudáveis para consumo diário (arroz integral, feijão, alface, couve, banana, beterraba, cenoura, maçã, tomate, uva, carne, peixe, frango e ovo) e 9 que, caso sejam consumidos diariamente, tornam-se nocivos à saúde (chocolate, batata chips, hamburguer, salsicha, balas, bolachas recheadas, pastel e refrigerantes). Além disso, considera-se saudável o consumo de 2L de água diários, ao 
invés de apenas $500 \mathrm{ml}$. O usuário deve assinalar apenas os alimentos considerados saudáveis. Após o jogo, o questionário foi reaplicado com o objetivo de avaliar se houve contribuição no processo de aprendizagem dos conceitos trabalhados no jogo.

Com o objetivo de levantar sugestões de melhoria, após o jogo, também foram coletados dados relacionados à satisfação da interação do usuário (Figura 2b). Buscou-se coletar as opiniões dos usuários relacionados com: a) design (contraste das cores, desenhos dos cenários e personagens); b) áudios (vocalização, trilhas sonoras e efeitos sonoros); c) interação (facilidade de navegar e facilidade de aprender a usar); d) diversão. Para coleta dessas informações, foi utilizada a escala de Likert com respostas variando entre Excelente, Muito bom, Bom, Razoável, Ruim e Não sei.

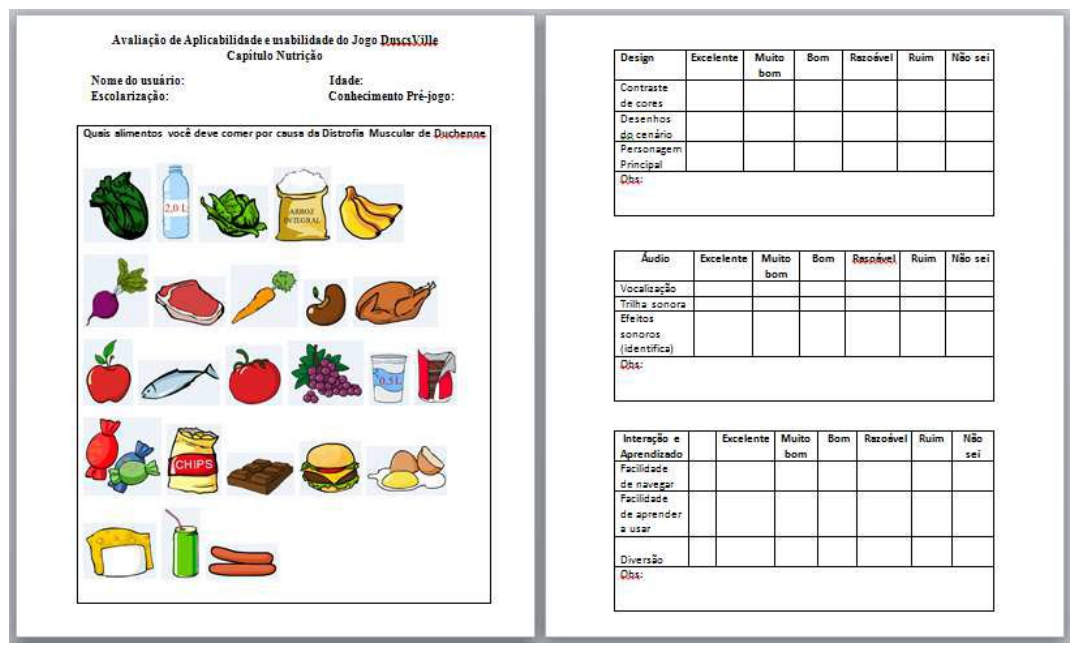

Figura 2 - a) Questionários de coleta de dados

\subsection{Resultados e Discussões}

O gráfico da Figura 3a mostra a proporção de acertos pré-jogo e pós-jogo coletados a partir do questionário de conhecimentos: "Quais alimentos você deve consumir diariamente para manter uma alimentação saudável?”. Percebe-se que, para a maioria dos usuários, houve um aumento de acertos após a interação com o jogo com taxa entre 0\% e 29\%. Em média, os usuários tiveram um aumento de 12\% de acertos considerando 9 usuários, pois o décimo usuário não quis responder ao questionário após o jogo.
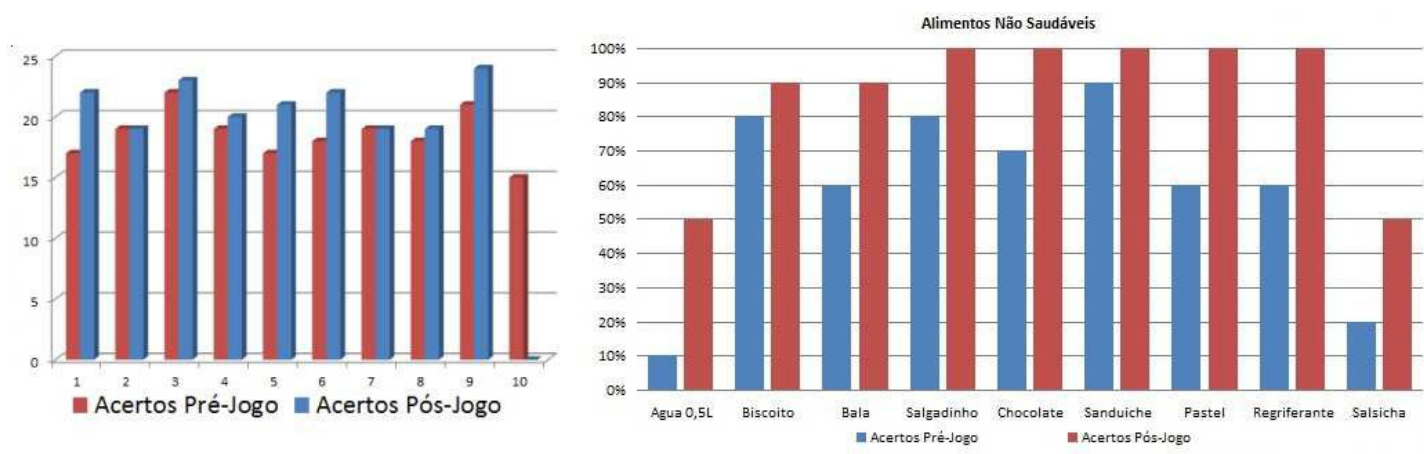

Figura 3 - a) Acertos pré-jogo e pós-jogo; b) Acertos alimentos não saudáveis

Observou-se que, no pré-jogo, os usuários já possuem conceitos sobre a importância de se consumir frutas, verduras e legumes, sendo esses os alimentos mais 
destacados nos questionários. O índice de acerto, para esses alimentos, permaneceu o mesmo após a aplicação do jogo. Já para alimentos tais como biscoito, balas, salgadinho, chocolate, sanduiche, pastel e salsicha, o índice de acerto foi baixo, antes da aplicação do jogo, e melhorou consideravelmente após o jogo (Figura 3b). De acordo com os profissionais da ABDIM, o consumo de alimentos tais como salsichas e linguiças são muito consumidos pelos pacientes, pois custam pouco dinheiro. As famílias atendidas são, em sua maioria, de baixo poder aquisitivo. Dessa forma, o consumo de alimentos baratos e não saudáveis é muito comum no dia a dia dos pacientes. Por este motivo, muitos pacientes acabam achando que esses alimentos são saudáveis, pois fazem parte da sua alimentação diária. Por isso, se torna relevante o desenvolvimento de ações educativas para que crianças e adolescentes com DMD participem da escolha dos alimentos para atingir o objetivo de controle de distúrbios nutricionais através do fortalecimento de um vínculo positivo entre educação e saúde.

Em relação à usabilidade, observa-se no gráfico da Figura 4 que o jogo teve uma boa aceitação pelo público-alvo, destaque para o design (desenhos, cenários e cores). A navegação, apesar de boa, ainda deixou a desejar, pois em algumas telas foi observado que o usuário não conseguia retroceder uma tela ou voltar ao desafio anterior. A trilha sonora e a vocalização também foram consideradas boas. O destaque nessa categoria ficou com os efeitos sonoros.

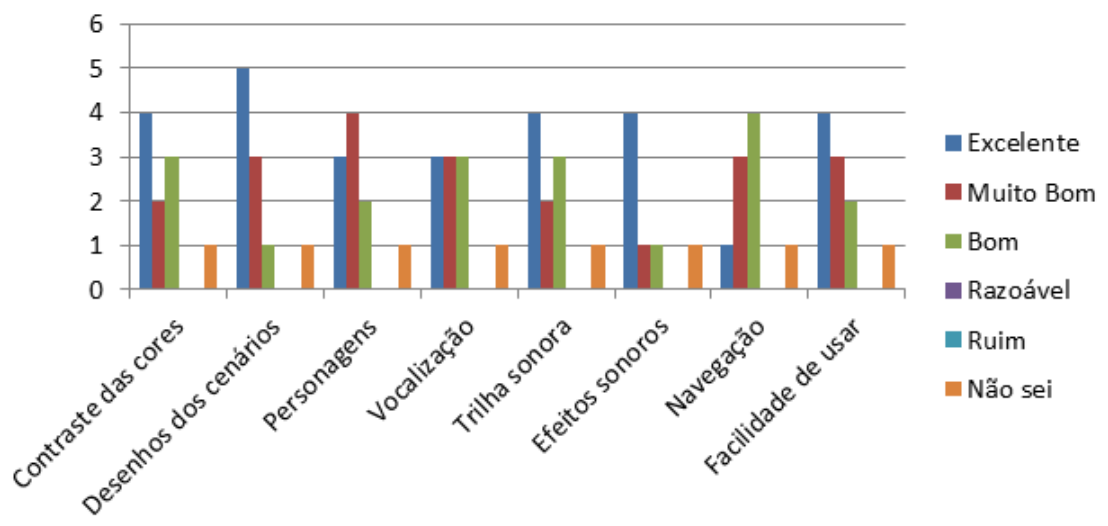

Figura 4 - Resultado do questionário de usablidade

Os usuários também opinaram sobre a interação com o jogo por meio de questões abertas. A coleta de comentários, expressos voluntariamente pelos usuários forneceu indícios sobre a aplicabilidade do jogo. Como exemplo cita-se um dos comentários coletados: “Gostei do joguinho, achei divertido. Errei porque peguei muitas comidas não saudáveis”.

\section{Conclusões}

Por meio deste estudo pode-se evidenciar que o desenvolvimento de um jogo educacional e sua disponibilidade pode contribuir para a intervenção nutricional com crianças com DMD, para promoção de saúde e mudanças nos hábitos alimentares das mesmas. A utilização do jogo DuchsVille, como fator motivacional é de fundamental importância, visto que esta tecnologia propiciou, de forma interativa, o contato com conceitos acerca da alimentação saudável, resultando em um maior interesse e participação do usuário pelo assunto abordado. 
No futuro, pretende-se ampliar este estudo no intuito de verificar se um grupo de usuários que utiliza o jogo regularmente por um período maior tem uma mudança efetiva do comportamento alimentar. Em caso positivo, serão consideradas alternativas de plataformas tecnológicas tais como TV Digital e telefones celulares o que permite novas formas de interação em espaços físicos diferentes, individualmente (telefones celulares) ou coletivamente (TV Digital).

\section{Referências}

BARANOWSKI, T.; BARANOWSKI, J.; CULLEN, K.W.; MARSH, T.; ZAKERI, I. Squire's Quest! Dietary outcome evaluation of a multimedia game. American Journal of Preventive Medicine, vol. 24, n.1, 2003, p. 52-61.

BARBOSA, L. Avaliação e adequação do Jogo Trilha Nutri Ação com Escolares da Rede Pública e Privada de Criciúma-SC. Trabalho de conclusão de Curso apresentado ao Curso de Nutrição da UNESC, Julho de 2010.

BIZZO, M.L.G; LEDER, L. Educação nutricional nos parâmetros curriculares nacionais para o ensino fundamental. Revistra Nutrição, vol. 18, n.5, 2005, p. 661-667.

COUTINHO, J.G., GENTIL, P.C.; TORAL, N. A desnutrição e obesidade no Brasil: o enfrentamento com base na agenda única da nutrição. Caderno de Saúde Pública, Rio de Janeiro, vol. 24, n.2, 2008, p. 332-340.

COSTA, A.G.V., CARBRINI, D., MAGALHÃES, R.D., FRANCESCHINI, S.C.; PIORE, S.E. Aplicação de jogo educativo para a promoção da educação nutricional de crianças e adolescentes. Revista Nutrição Brasil, São Paulo, vol. 3, n.4, 2004, p. 205-209.

DAVIDSON, Z.E. \& TRUBY, H. A review of nutrition in Duchenne muscular dystrophy. Journal of Human Nutrition and Dietetics, vol. 22, 2009, pp. 383-393.

HANKARD, R.; GOTTRAND, F.; TURCK, D. Resting energy expenditure and energy substrate utilization in children with Duchenne muscular dystrophy. Pediatric research, vol 40, 1996, p. 29-33.

JORGE, T.C.; PERES, S.P.B. Elaboração de recursos pedagógico-nutricionais para o programa de educação nutricional. Revista Nutrição Brasil, São Paulo, vol. 3, n.4, 2009, p. 211-218.

MAGALHÃES, C.R. O jogo como pretexto educativo: educar e educar-se em curso de formação em saúde. Revista Interface, Comunicação, Saúde e Educação, vol. 11, n.23, 2007, p. 647-654.

MANZUR, A.Y.; KINALI, M.; MUTONI, F. Up date on the management of Duchenne muscular Dystrophy. Archives of disease in childhood, vol. 93, 2008, p. 986-90.

MATHIAS, C.V.; NUNES, J.F. FAGAN,S.B. Objetos de Aprendizagem na Educação Infantil. Revista Novas Tecnologias na Educação (RENOTE), vol. 7, n.1, 2008.

MAYO, A.L.; CRAVEN, B.C.; MACADAM, L.C.; BIGGAR, W.D. Bone health in boys with Duchenne Muscular Dystrophy on long-term daily deflazacort therapy. Neuromuscul Disord, vol 22, n.12, 2012, p. 1040-1045. 
NEVES, A.S. Desenvolvimento de Software: Sistema Informatizado de Educação Nutricional via Web. Dissertação apresentada ao Curso de Mestradoem Ensino em Ciências e Saúde e do Meio Ambiente do UniFOA, 2010.

PANE, M.; VASTA, I.; MESSINA, S.; SORLETTI, D.; ALOYSIUS, A.; SCIARRA, F.; MANGIOLA, F.; KINALI, M.; MERCURI, E. Feeding problems and weight gain in Duchenne muscular dystrophy”. European journal of paediatric neurology, vol. 10, 2006, p. 231-236.

PREECE, J.; ROGERS, Y.; SHARP, H. Design de Interação: Além da Interação Homem Computador. USA: Bookman, 2005, 348 p.

SANTOS, N.M.; REZENDE, M.M.; TERNI, A.; FÁVERO, F.M.; QUADROS, A. J.; LANGER, A.N.; FONTES, S.V.; OLIVEIRA, A.S.B. Perfil clínico e funcional dos pacientes com Distrofia Muscular de Duchenne assistidos na Associação Brasileira de Distrofia Muscular (ABDIM). Revista Neurociências, vol.14, n.1, 2006, p. 15-22.

SANTOS, L.A.P. Educação alimentar e nutricional no contexto da promoção de práticas alimentares saudáveis. Revista Nutrição, vol.18, n.5, 2005, p. 681-692.

Standing Committee on Nutrition. Diet-related chronic diseases and double burden of malnutrition in West Africa. London: United Nations System, n. 33, 2006.

SAVI, R.; ULBRICHT, V.R. Jogos Digitais Educacionais, Benefícios e Desafios. Revista Novas Tecnologias na Educação (RENOTE), vol. 6, n.2, 2008.

TOSCANI, N.V.; SANTOS, A.J.; SILVA, L.M.; Tonial, C.T.; Mezzari, A. Desenvolvimento e análise de jogo educativo para crianças visando à prevenção de doenças parasitológicas”. Revista Interface, Comunicação, Saúde, Educação, vol. 11, n.22, 2007, p.281-294.

VECHIA, L.D. Avaliação do Estado Nutricional d Portadores de Distrofia Muscular Vinculados à Associação Sul Catarinense de Amigos, familiares e Portadores de Distrofias Musculares Progressivas da Região de Criciúma. Trabalho de Conclusão de Curso apresentado ao Curso de Nutrição da UNESC. Julho de 2009.

ZACCARELLI, E.M.; Coelho, H.D.S.; Silva, M.E.P. O jogo, como prática educativa, no treinamento para controle higiênico-sanitário, em unidades de alimentação e nutrição. Revista Higiene Alimentar, vol. 14, n.70, 2000 p.23-26. 